\title{
Multimodal imaging system for dental caries detection
}

\author{
Rongguang Liang, Victor Wong, Michael Marcus, Peter Burns, and Paul McLaughlin \\ Eastman Kodak Company \\ 1700 Dewey Avenue, Rochester, NY 14650-1816
}

\begin{abstract}
Dental caries is a disease in which minerals of the tooth are dissolved by surrounding bacterial plaques. A caries process present for some time may result in a caries lesion. However, if it is detected early enough, the dentist and dental professionals can implement measures to reverse and control caries. Several optical, nonionized methods have been investigated and used to detect dental caries in early stages. However, there is not a method that can singly detect the caries process with both high sensitivity and high specificity. In this paper, we present a multimodal imaging system that combines visible reflectance, fluorescence, and Optical Coherence Tomography (OCT) imaging. This imaging system is designed to obtain one or more two-dimensional images of the tooth (reflectance and fluorescence images) and a threedimensional OCT image providing depth and size information of the caries. The combination of two- and threedimensional images of the tooth has the potential for highly sensitive and specific detection of dental caries.
\end{abstract}

Keywords: dental caries, multimodal imaging, reflectance, fluorescence, optical coherence tomography

\section{INTRODUCTION}

Dental caries is an infectious, communicable disease resulting in destruction of tooth structure by acid-forming bacteria found in dental plaque, an intraoral biofilm, in the presence of sugar. The infection results in the loss of tooth minerals that begins with the outer surface of the tooth and can progress through the dentin to the pulp, ultimately compromising the vitality of the tooth. The early detection and characterization of caries lesions is very important because surgical restorative procedures could be reduced. If detected at an early stage, the dentist and dental professionals can implement measures to reverse and control caries, such as identifying patients in need of preventive care, implementing fluoride treatments, implementing plaque control measures, and identifying patients that are at high risk of developing dental caries.

As has been investigated, the loss of mineral from the enamel and dentin is known to alter the optical properties of teeth, such as scattering, reflection, absorption, and fluorescence. Light is a particularly suitable tool to study tooth decay. Structural disruption and the uptake of fluid into pores caused by demineralization change the normal interaction of light with tooth structure. These changes include scattering, absorption, and fluorescence. Other changes, such as exogenous stain, bacterial breakdown products, and other contaminants present as a result of the caries process also can be detected by optical methods.

Many optical technologies have been developed to characterize the changes of optical properties of tooth structure related to demineralization. Those technologies include fluorescence, fiber-optic trans-illumination, confocal scanning, Optical Coherence Tomography (OCT), photothermal radiometry, structural light illumination microscopy, and multiphoton microscopy. All of these methods characterize one or more property changes of the tooth structure.

Fluorescence technique has been widely used in biomedical imaging since the phenomenon of fluorescence was investigated by English physicist Stokes in $1852 .{ }^{1}$ The fluorescence of teeth was discovered by Stubell in 1911 with UV excitation. $^{2}$ In the early $1980 \mathrm{~s}$, Alfano and Yao reported the first systematic investigation of tooth fluorescence with different light excitations. ${ }^{3,4}$ They discovered that by using light of wavelength $350 \mathrm{~nm}, 410 \mathrm{~nm}$ and $530 \mathrm{~nm}$, the emission wavelength peaks obtained for teeth were, respectively, $427 \mathrm{~nm}, 480 \mathrm{~nm}$, and $580 \mathrm{~nm}$. The peaks of the emission spectra of carious regions were shifted to the red portion of the spectrum. Also, Alfano and Yao reported that the relative intensity of light in the red portion was greater for a carious region than for a sound region. Two fluorescence techniques have been developed to detect dental caries. The first technology with blue light excitation, Quantitative Laser Fluorescence (QLF), was developed in Sweden and Holland. ${ }^{5,6}$ When teeth are illuminated with high-intensity blue light, they will emit light through the visible spectrum. Sound, healthy enamel shows a higher fluorescence than demineralized enamel; demineralized areas appear relatively darker under light that excites the fluorescence. The amount of mineral loss in surface lesions correlates strongly with the loss of fluorescence signal. This technique is 
commercialized by Inspektor Research Systems BV, NL. Another technology, called Laser Fluorescence (LF), with red light excitation, was developed by Hibst and Gall in $1998 .^{7}$ When teeth are illuminated with red light, the fluorescence radiation from healthy tooth regions is strongly reduced so that the fluorescence radiation from carious regions is superposed with autofluorescence of the healthy tooth tissue. Clean, healthy tooth structure exhibits little or no fluorescence. Red fluorescence is proportional to the severity of tooth decay. This technique is developed and commercialized by KaVo Dental GmbH.

Optical coherence tomography (OCT) is an interferometric, noninvasive optical tomographic imaging technique offering millimeter penetration with micrometer axial and lateral resolution. The technique was first demonstrated at MIT in 1991. With micrometer resolution and cross-sectional imaging capabilities, OCT has become a prominent biomedical tissue imaging technique. Dental OCT imaging was pioneered by a collaboration between Lawrence Livermore National Laboratory and the University of Connecticut in $1995 .{ }^{8,9}$ Preliminary work focused on correlating optical scattering signatures with tissue structures in the periodontium region of the oral cavity. Since then, several groups have investigated OCT imaging in dental applications. In 2002, B. Amaechi at the University of Texas at San Antonio observed a linear correlation between the mineral loss in enamel and dentine measured by transverse microradiography (TMR) and the reflectivity loss measured by OCT. ${ }^{10}$ In 2005 , Fried demonstrated that the increase in the integrated reflectivity in the PS-OCT perpendicular polarization axis correlates well with increasing mineral loss/severity caused by natural demineralization, by comparing with digital microradiographs (DM) and polarized light microscopy (PLM) ${ }^{11} \mathrm{He}$ suggested that it is necessary to acquire images in both polarization states in order to identify areas of demineralization, remove the confounding influence of the strong reflection at the tooth surface, and quantify the lesion severity.

While QLF and LF have good sensitivity in the detection of dental caries, both techniques have limitations. For example, both QLF and LF do not provide information about the lesion depth and suffer from low specificity attributed to the presence of plaque, calculus, and/or staining on the tooth surface. In addition, ambient light and hydration of tooth tissue also degrade the quality of the QLF image. Using a NIR $1310 \mathrm{~nm}$ light source, OCT imaging can provide high-resolution tooth images with depth, size, and boundary information. However, the OCT system has a small field of view and is not practical for large-scale imaging for in-vivo dental applications.

In order to detect dental decay with high sensitivity and specificity, we have developed a multimodal imaging system with the combination of reflectance, fluorescence, and OCT imaging. Fluorescence and OCT images are distinct and complementary, with fluorescence providing information about biochemical composition and OCT information about tooth structural information. With the help of reflectance imaging, calculus, stain, and amalgam can be clearly shown. Thus, the combination of reflectance, fluorescence, and OCT imaging modalities may enable higher sensitivity and specificity for dental caries detection than any single modality alone.

In the system we have developed, the wide-field-of-view reflectance and fluorescence images are used to identify regions of interest for OCT imaging. The large areas can be screened quickly with reflectance and fluorescence imaging; only regions that have abnormal fluorescence are investigated in detail with OCT.

\section{MULTIMODAL IMAGING SYSTEM}

Figure 1 shows the diagram of the multimodal imaging system that we have developed. There are two subsystems in this diagram. One is the area imaging subsystem to capture reflectance and fluorescence images; another is the OCT imaging system. Two light sources (LS) are used for area images. A blue light source is used to excite fluorescence and a white light source is used to obtain the reflectance image. The two light sources are combined by a dichroic mirror, which reflects light from the white light source and transmits light from the blue light source. In order to remove specular reflection, the polarized illumination method is applied in this system. A polarization beamsplitter (PBS) is used as a polarizer to condition the illumination light so that linearly polarized light illuminates the tooth surface. After the light reflects and scatters back from the tooth, the polarization beamsplitter works as an analyzer to block the light with the same polarization state as the illumination beam, but transmits the light with the orthogonal polarization state to the sensor. Also, in order to block the blue excitation light, a long pass filter (LP) is placed in the optical path before the sensor. This filter should have a sharp cutoff edge and very good extinction to block the blue excitation light while transmitting visible light, so that the system can capture visible reflectance images without having to move the LP filter out of the optical path. In order to integrate with an OCT imaging system, another dichroic mirror is used to direct the light from the OCT subsystem to the tooth surface. As shown in Figure 1, the dichroic mirror reflects the infrared light from the OCT subsystem and transmits visible light from the area imaging subsystem. The OCT subsystem can be a time-domain or Fourier-domain system. 
This multimodal imaging system has the advantage that the user can screen the tooth with visible illumination light to obtain a visible reflectance image, just as a conventional intra-oral camera, or screen the tooth with blue excitation light to view the fluorescence image. With the white light reflectance image and the fluorescence image, and possibly with further image processing, the user can identify the healthy, carious, and suspicious regions. Once the carious and suspicious regions are located, OCT imaging can then be used to scan those regions. For carious regions, OCT images can provide more detailed information, such as decay depth, size, and boundary. For suspicious regions, OCT images can verify whether the regions are indeed carious lesions, and if so, how deep the lesions are. All three images, reflectance, fluorescence, and OCT, can be saved for progression monitoring.

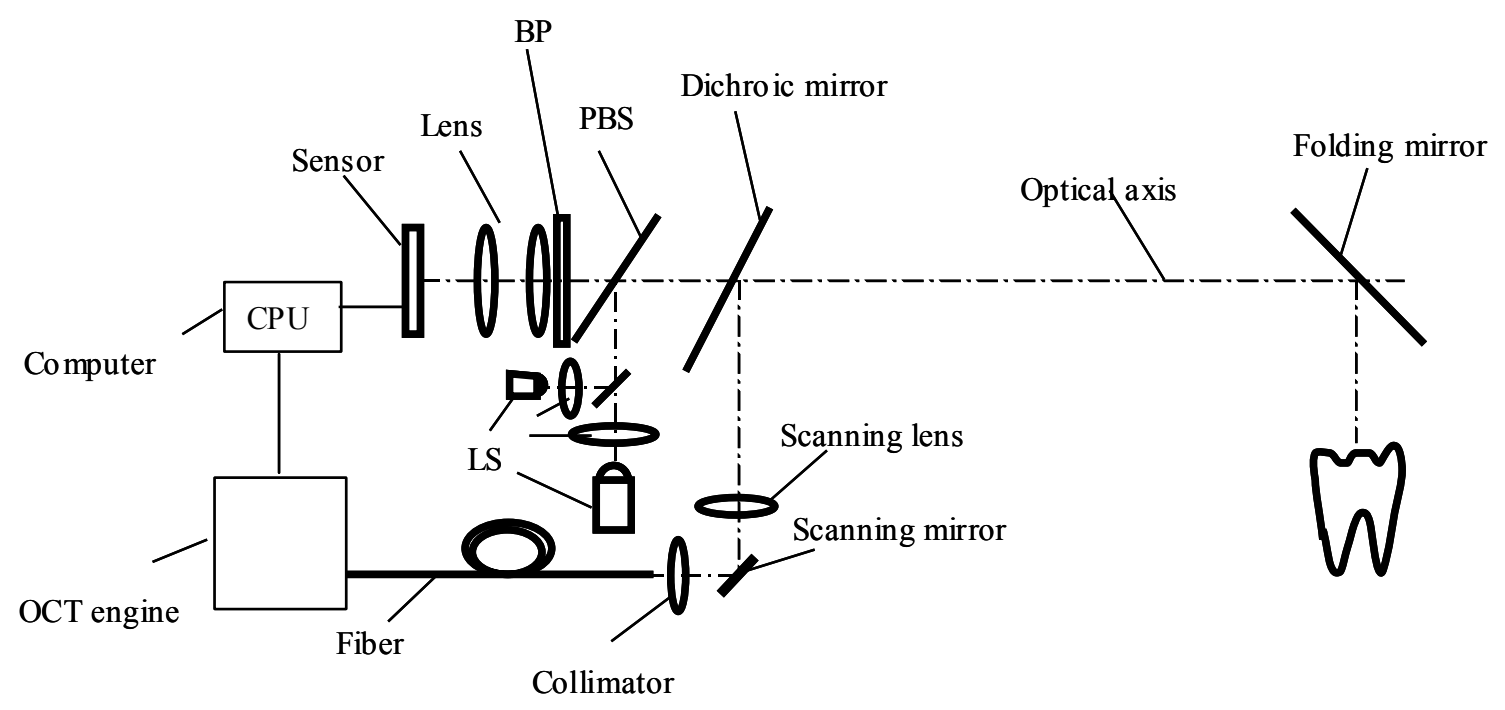

Fig. 1. Diagram of the multimodal imaging system.

\section{EXPERIMENTAL RESULTS}

To test the performance of the multimodal imaging system for dental caries detection, we have built a prototype and evaluated its performance on extracted tooth samples. After the teeth were extracted, they were placed in a fresh solution of Chloramine T $0.5 \%$ for three days for sterilization. Some tooth samples were sectioned so that we can correlate OCT images with the tooth structural information beneath the tooth surface. For each tooth sample, the imaging system captured and saved all three images: visible reflectance, fluorescence, and OCT. For the sectioned teeth, the OCT image is taken scanning along the cutting edge of the tooth.

Figure 2 shows the three images obtained with the multimodal system on the occlusal surface of a sectioned tooth, along with an image of the tooth section. All three images clearly show there is a carious region: white spot in the reflectance image (Figure 2(a)), dark area in the fluorescence image (Figure 2(b)), and the scattering profile in the OCT image (Figure 2(d)). The carious region is also verified by the tooth section (black box in Figure 2(c)).

Figure 3 demonstrates a case in which OCT is able to identify false positives in the reflectance and fluorescence images. The white spot in the visible reflectance image (Figure 3(a)) and the dark region in the fluorescence image (Figure 3(b)) both indicate the presence of caries. However, the OCT image shown in Figure 3(d) does not show much scattering beneath the tooth surface, which means there is very little decalcification. This finding is validated by the digital image of the tooth section (white box in Figure 3(c)), which shows just the beginning stage of decalcification. The OCT image in Figure 3(d) also shows the difference between white spot (stronger signal due to higher reflection) and the healthy region even when the decalcification is very shallow. 


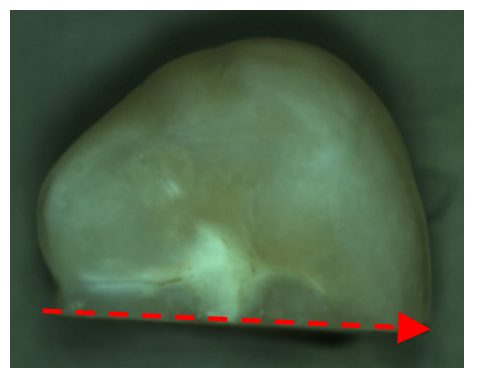

(a)

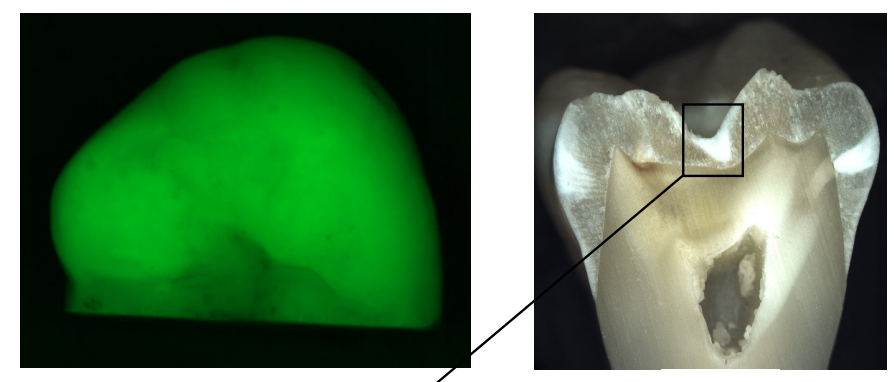

(b)

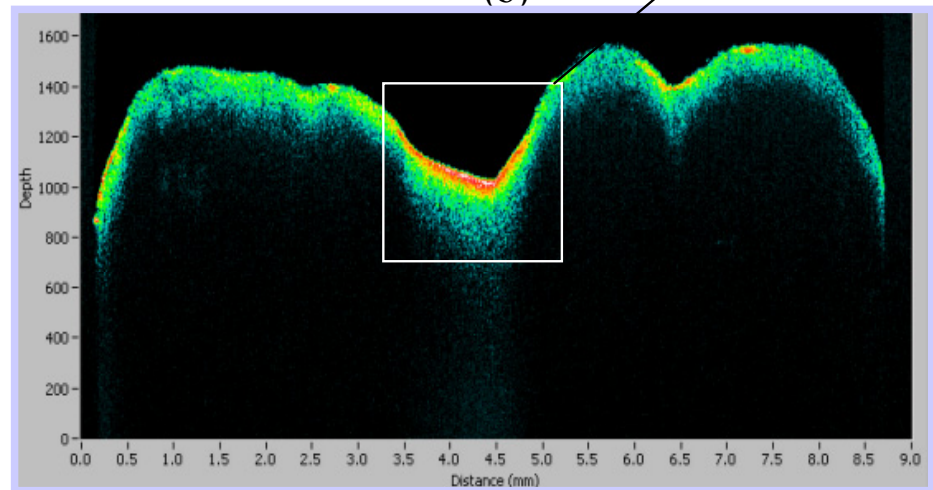

(d)

(c)

Fig. 2. Visible reflectance (a), fluorescence (b), and OCT (d) images of the occlusal surface of a sectioned tooth. (c) is the image of the tooth section. The dotted arrow shows the OCT scan line and direction. The caries captured by OCT (white box in (d)) correlates well with that in the section image (black box in (c)).

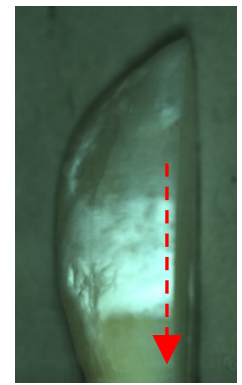

(a)

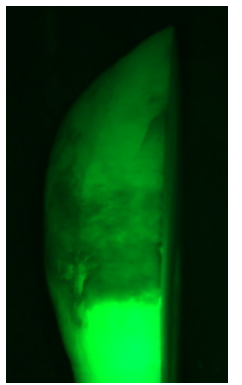

(b)

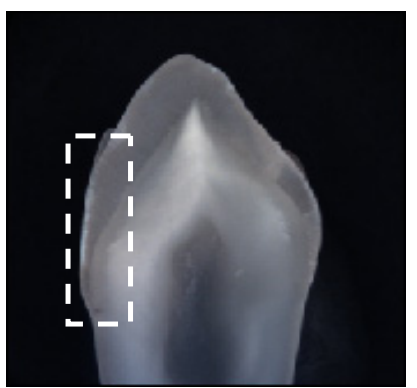

(c)

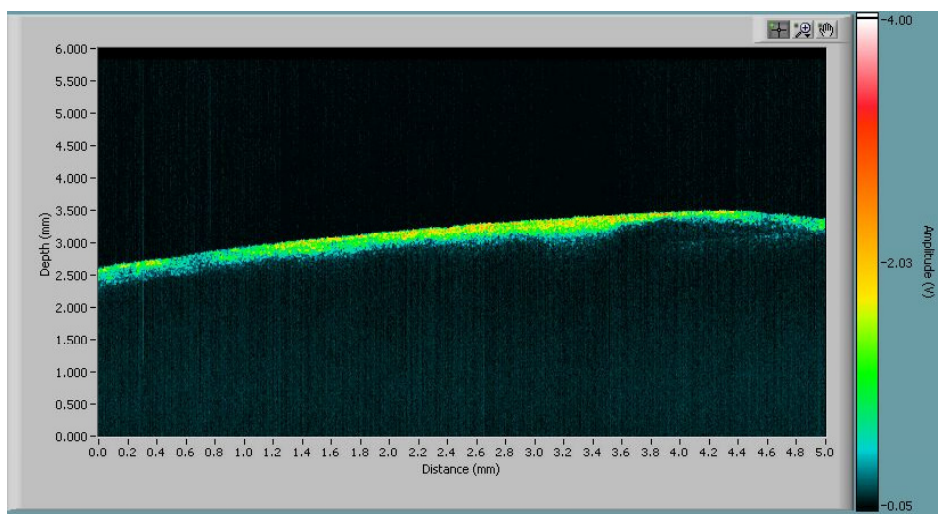

(d)

Fig. 3. Reflection (a), fluorescence (b), and OCT images (d) of a sectioned tooth. The image of the tooth section is displayed in (c). The dotted arrow indicates the OCT scan position and direction. 
Figure 4 presents a different scenario where the visible image shows clearly that there is a carious region along the cutting edge (white spot in Figure 4(a)). However, the caries is not shown in the fluorescence image in Figure 4(b). The OCT image in Figure 4(d) verifies that the region is indeed carious. The caries is shown very clearly in the image of the tooth section in Figure 4(c). This example shows that the combination of OCT and fluorescence images can be used to increase the sensitivity of caries detection.

Unfortunately, stain, calculus, and other debris on the tooth surface can impair the specificity of fluorescence imaging in caries detection. Figure 5 is an illustration that stain causes false positive in the fluorescence image. As shown in Figure 5(a), there is stain on the tooth surface, and the stain appears darker in the fluorescence image in Figure 5(b). If only a fluorescence image is available, these darker regions in the fluorescence image may be misinterpreted as caries. With the OCT image, it is clear that there is no decalcification beneath those regions.

OCT can also identify areas with abnormal fluorescence. In Figure 6, the reflectance image shows a large white spot, but the corresponding region in the fluorescence image is not darker, as this is how caries are generally manifested in the fluorescence image. Instead, the area corresponding to the white spot is slightly brighter in the fluorescence than in the surrounding area. This abnormal fluorescence effect is misleading; the cause of the effect is not clear. The OCT image in Figure 6(c) shows that the white spot shown in the reflectance image has a similar scattering signature as decalcification.

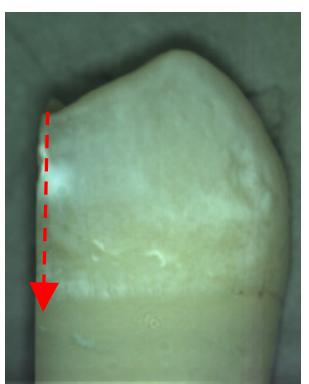

(a)

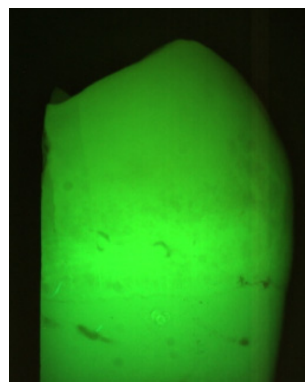

(b)

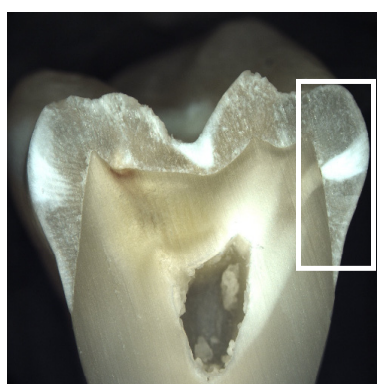

(c)

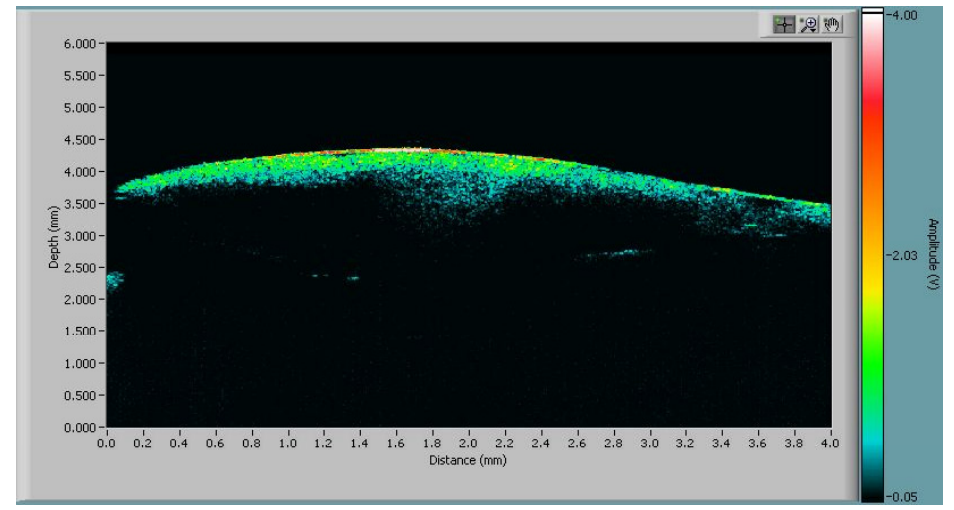

(d)

Fig. 4. Reflection (a), fluorescence (b), and OCT images (d) of a sectioned tooth. The image of the tooth section is displayed in (c). The dot arrow indicates the OCT scanning position and direction.

\section{SUMMARY}

We have developed and tested a multimodal imaging system to detect early dental caries with potentially high sensitivity and specificity. This system integrates visible reflectance, fluorescence, and OCT imaging capabilities. Three imaging modalities correlate with each other and/or complement each other in detecting caries, stain, calculus, and other property changes of tooth structure. 


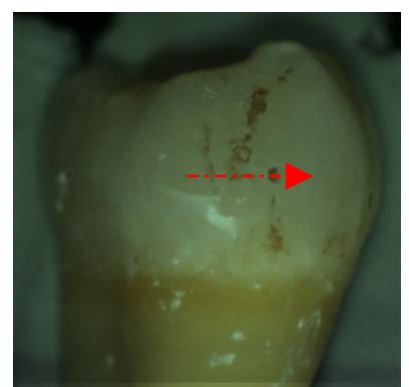

(a)

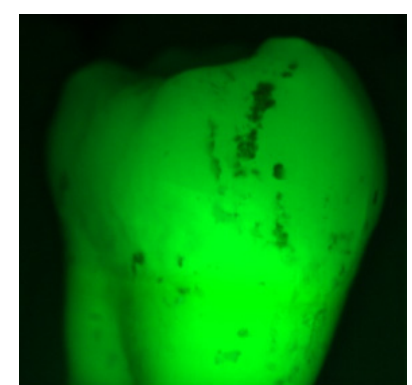

(b)

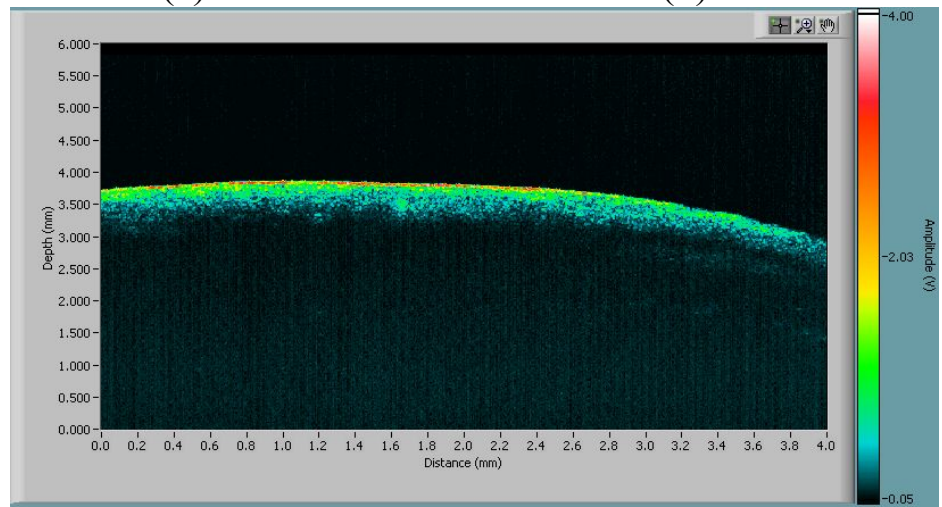

(c)

Fig. 5. Reflection (a), fluorescence (b), and OCT images (c) of a tooth with stain. The dot arrow indicates the OCT scanning position and direction.

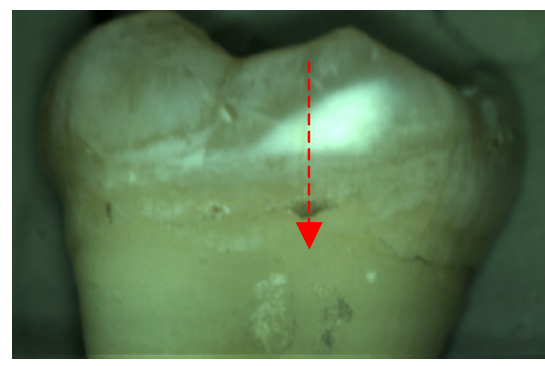

(a)

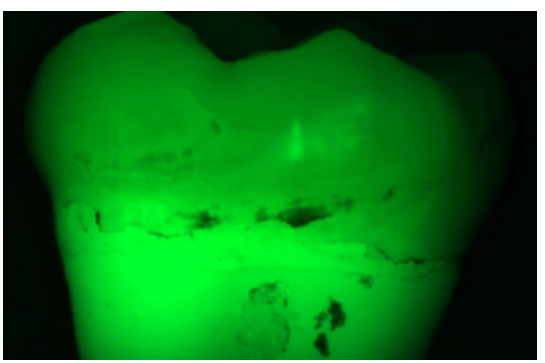

(b)

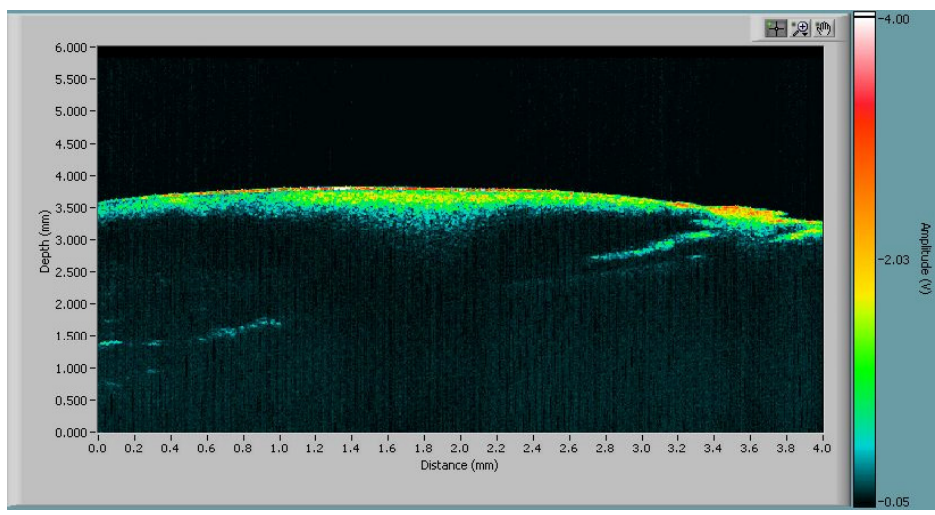

(c)

Fig. 6. Reflection (a), fluorescence (b), and OCT images (c) of a tooth. The dot arrow indicates the OCT scanning position and direction. 
With wide-field-of-view visible reflectance and fluorescence imaging, the carious and suspicious regions can be easily identified, and the detail information of those regions can be further obtained by OCT scanning. While we have evaluated its performance, we will further develop image-processing algorithms to improve image contrast, image registration, and feature identification to allow the system to detect dental caries automatically and monitor the progression of the suspicious regions.

\section{ACKNOWLEDGMENT}

We thank our colleagues, Mark Bridges, Laurie Voci, and Gary Spinelli for helpful discussions of this and related work.

\section{REFERENCES}

1. G.Q. Stokes, "On the change of refrangibility of light." Phil Trans Roy Soc London, A142, 463, 1852.

2. H. Stubell, "Die fluoreszone tierische gewebe in ultra-violettuin licht." PflingersArch ges Physiol, 141, 1-14, 1911.

3. R.R. Alfano and S.S. Yao, "Human teeth with and without dental caries, studied by visible luminescent spectroscopy." J Dent Res 54, 67, 1981.

4. R.R. Alfano and S.S. Yao, "Human teeth with and without caries studied by laser scattering, fluorescence and absorption spectroscopy,” IEEE Journal of Quantum Electronics, 20, 1512-1516, 1984.

5. H. Bjelkhagen, F. Sundstrom, B Angmar-Manson and H Ryden, "Early detection of enamel caries by luminescence excited by visible laser light." Swed Dent J 6, 1-7, 1982.

6. E. de Josselin de Jong, F. Sundstrom, U Hafstrom-Bjorkman and B Angmar-Manson, "A new method for the longitudinal measurement of mineral changes in enamel: Quantitative laser fluorescence." Caries Res 25, 215, 1991.

7. R. Hibst and R. Gall, "Development of a diode laser-based fluorescence caries detector." Caries Res 32, 294, 1998.

8. B.W. Colston, "OCT for diagnosis of periodontal disease." Proceedings of SPIE 3251, p52-58, 1998.

9. B.W. Colston, "Dental OCT." Opt. Express 3, 230-238, 1998.10.

10. B.T. Amaechi, "Optical coherence tomography for dental caries detection and analysis." Proceedings of SPIE 4610, 100-108, 2002.11.

11. D. Fried, "Imaging caries lesions and lesion progression with polarization sensitive optical coherence tomography." J Biomed Opt 7(4), 618-627, 2002.12.

12. B. Amaechi, "Correlation of quantitative light-induced fluorescence and optical coherence tomography applied for detection and quantification of early dental caries.” J Biomed Opt 8(4), 642-647, 2003. 